\title{
Failure of Laparoscopic Sleeve Gastrectomy - Further Procedure?
}

\author{
Rudolf A. Weiner Sophia Theodoridou Sylvia Weiner \\ Department of Surgery, Krankenhaus Sachsenhausen, Frankfurt/M., Germany
}

\section{Keywords}

Sleeve gastrectomy - Failure in treatment . Weight regain - Revisional surgery

\section{Summary}

Background: Worldwide, the incidence of morbid obesity is increasing, and surgery is the only effective longterm treatment. Laparoscopic sleeve gastrectomy (LSG) is associated with acceptable weight loss and reduced comorbidities. It is considered a safe procedure with sporadic complications. This publication aims to describe failures of LSG in terms of ineffective weight loss or early weight regain, and analyze secondary treatment options. Methods: From October 2001 to December 2010, 937 patients underwent LSG in our department. Initially, all procedures were scheduled as a two-stage procedure (LSG followed by biliopancreatic diversion with duodenal switch). However, the second procedure was not performed in 64 patients (body mass index $>60 \mathrm{~kg} / \mathrm{m}^{2}$ ). Since 2005, the frequency of second stage procedures after weight regain has been increasing; their outcome is analyzed. Results: Of the 937 patients, 17 (1.8\%) experienced staple line leakage. Mean time to first re-intervention or endoscopic stent placement was $15.6 \pm 22$ days (range 2-78). From 2005 to 2010, 106 secondary procedures were performed. Insufficient weight loss or weight regain were the indications in 88 cases. Sixteen (15\%) patients had severe gastroesophageal reflux which was resolved by Roux-en-Y gastric bypass (RYGB). Stenosis was observed in $2(2.6 \%)$ patients, which required endoscopic dilatation and stent placement in one case and gastric bypass in the other. Conclusions: LSG is a feasible and popular bariatric procedure. Mortality $(0.4 \%)$ was much higher than after gastric bypass $(0.03 \%)$ and gastric banding $(0 \%)$ The knowledge of potential complica- tions and their management is crucial. All restrictive procedures require patient compliance, but increased food uptake after RYGB and LSG is common. Malabsorptive procedures are more effective for long-term weight loss. Duodenal switch and omega-loop gastric bypass are more efficient second stage procedures than re-sleeve or RYGB.

\section{Introduction}

Obesity surgery has become more common due to the worldwide obesity epidemic. A shift from open to laparoscopic surgery has occurred in the 1990s because of its advantages. The distribution of standard procedures has changed since the beginning of modern obesity surgery, but the basic principles of restriction and malabsorption are still the same. Hormonal regulation is under investigation and has shown complex results, but the effects on appetite, hunger, and weight loss are not completely clear. The prevalence and selection criteria for different procedures are changing. The main changes have occurred in relation to restrictive procedures during the last 2 decades. Three restrictive procedures are currently in use: adjustable gastric banding, laparoscopic sleeve gastrectomy (LSG), and proximal gastric bypass. Malabsorptive procedures like biliopancreatic diversion with or without duodenal switch (BPD or BPD-DS) and distal gastric bypass are still not the first choice for the majority of surgeons. In the US, restrictive procedures represent more than $95 \%$ of all surgeries. In contrast to BPD, BPD-DS and distal bypass, all restrictive procedures show significant weight regain in the mid and long term. This situation is not a disaster because overweight

\section{KARGER \\ Fax +497614520714 \\ Information@Karger.de}

www.karger.com (c) 2011 S. Karger GmbH, Freiburg

$1662-4025 / 11 / 0047-0042 \$ 38.00 / 0$

Accessible online at:

www.karger.com/ofa
Prof. Dr. med. Rudolf A. Weiner

Chirurgische Klinik, Krankenhaus Sachsenhausen

Schulstraße 31, 60594 Frankfurt, Germany

Tel. +49 69 6605-1131, Fax -1203

rweiner@khs-ffm.de 


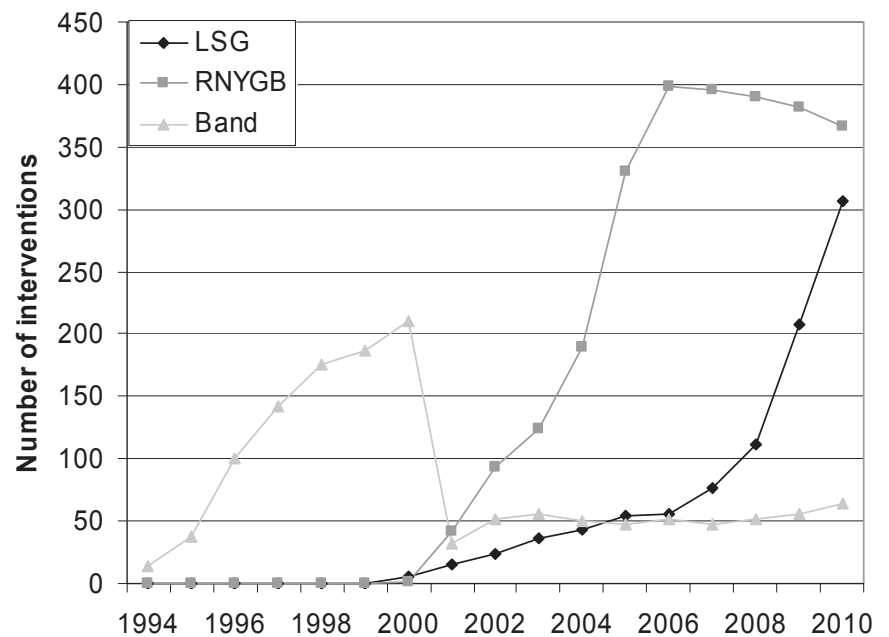

Fig. 1. Number of standard procedures (gastric banding, gastric bypass, sleeve gastrectomy) during the last 17 years.

and comorbidities are still reduced for 10-15 years, which will prolong overall life expectancy. However, patients are reacting to this situation more than physicians. Revisional surgery after procedures for weight loss in morbidly obese patients is becoming an important issue, and restrictive procedures account for a large proportion of these interventions. All have proven effective, but less so than malabsorptive or combined procedures. The reasons for re-operation and conversion to a different procedure are failure of the operation in terms of insufficient weight loss or weight regain (secondary obesity), or complications such as penetration, infection, bleeding, obstruction, dysphagia, and gastroesophageal reflux (GERD). This paper will describe the concept of failed LSG leading to a second operation, the conditions and potential complications present after failed LSG, the technical steps required, and the outcome of revisional surgery.

\section{Patients and Methods}

A retrospective analysis of patients who underwent primary LSG and laparoscopic revisional bariatric surgery for complications after previous LSG from 2005 to August 2010 was performed. In this period, $937 \mathrm{pa}-$ tients underwent LSG. In the first 125 cases (mean BMI $64.4 \pm 9.3 \mathrm{~kg} / \mathrm{m}^{2}$ ), BPD-DS was intended as second stage procedure in high-risk superobese patients, but 64 of these patients did not undergo this procedure within the following 2 years. In the last 5 years (mean BMI $58.5 \pm 7.3 \mathrm{~kg} / \mathrm{m}^{2}$ ), LSG was intended as a definitive procedure. The sleeve size changed during this period. For the first stage of BPD-DS, the antrum was preserved and the diameter of the calibration tube was 54-60 French. Later, the calibration tube was 32 French, and after an increase in staple line leaks the size was changed to 42 French. Staple line reinforcement was performed in two thirds of all procedures. Reinforcement of the antrum by oversewing was performed routinely. Technical revisions included silicone-ring placement, re-sleeve gastrectomy, conversion to Roux-en-Y gastric bypass (RYGB), omega-loop gastric bypass, and BPD-DS. The data collected included pre- and postoperative BMI, operating time, blood loss, length of hospital stay, and intraoperative and postoperative complications.

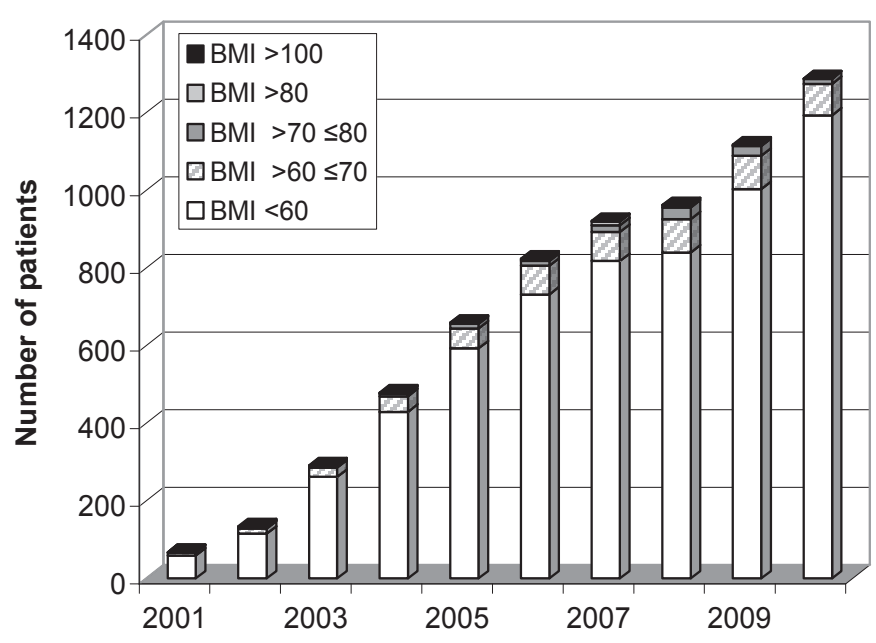

Fig. 2. BMI classes of all patients for obesity surgery from 2011 to 2010 in the Krankenhaus Sachsenhausen Frankfurt am Main (Germany).

\section{Results}

The number of procedures carried out in our department has been rapidly increasing during the last few years (fig. 1). There was a relative and absolute increase in LSG. An indication for multiple-step treatments in patients with a BMI of $>60 \mathrm{~kg} / \mathrm{m}^{2}$ still arises in Germany. Figure 2 shows the BMI distribution of operated patients in our department. Overall, 925 LSG were completed without conversion. In 2 cases, the procedure was stopped due to extreme liver enlargement. Following conservative intragastric balloon treatment, LSG was performed 4 and 6 months later. The operating time shows a wide variation from $25 \mathrm{~min}$ up to $170 \mathrm{~min}$. The number of different surgeons during the time period of 10 years was 9. Bleeding was the most common complication: 46 patients lost more than $800 \mathrm{ml}$ of blood; blood transfusions were necessary in 12 patients; 8 patients underwent re-laparoscopy within $24 \mathrm{~h}$; in 2 cases an active bleed was stopped by suture; in 6 patients evacuation of the abdominal cavity and demonstration of non-active bleeding was the only management. Staple line leakage was observed in $17(1.8 \%)$ patients as the main complication. The mean time from first surgery to first re-intervention or endoscopic stent placement was $15.6 \pm$ 22 days (range 2-78 days). From 2005 to 2010, we performed 106 secondary procedures after LSG. In 76 patients LSG was carried out in our hospital, and 30 patients underwent the procedure in another institution. Insufficient weight loss or weight regain $(<50 \%$ excess weight loss, EWL) was the indication for a secondary procedure in 88 cases. In more than $50 \%$, anatomically incorrect sleeves were seen (fig. 3-5). In 16 (15\%) patients severe GERD was seen after LSG, which was resolved by RYGB; 9 patients showed GERD from the beginning, and 7 patients developed severe GERD more than 1 year after LSG. Stenosis was observed in $3(3.9 \%)$ patients of whom $2(2.6 \%)$ were re-operated. In one of the 3 patients, the stenosis was located in the gastroesophageal junction; in 


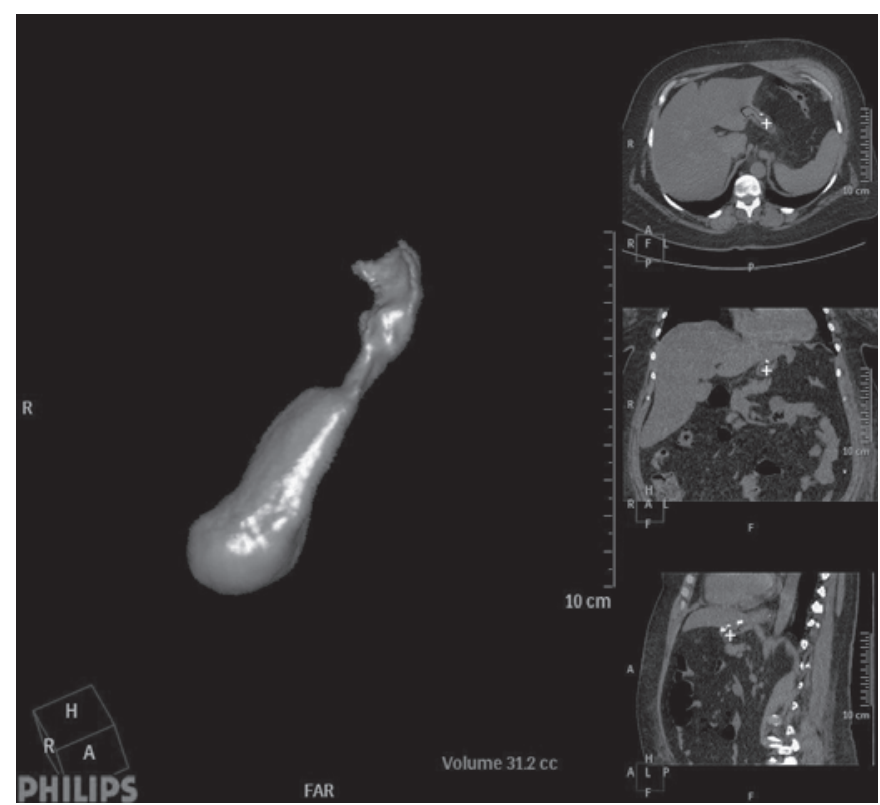

Fig. 3. Virtual $\mathrm{CT}$ after sleeve gastrectomy 3rd postoperative day: normal finding after calibration with 42 French tube.

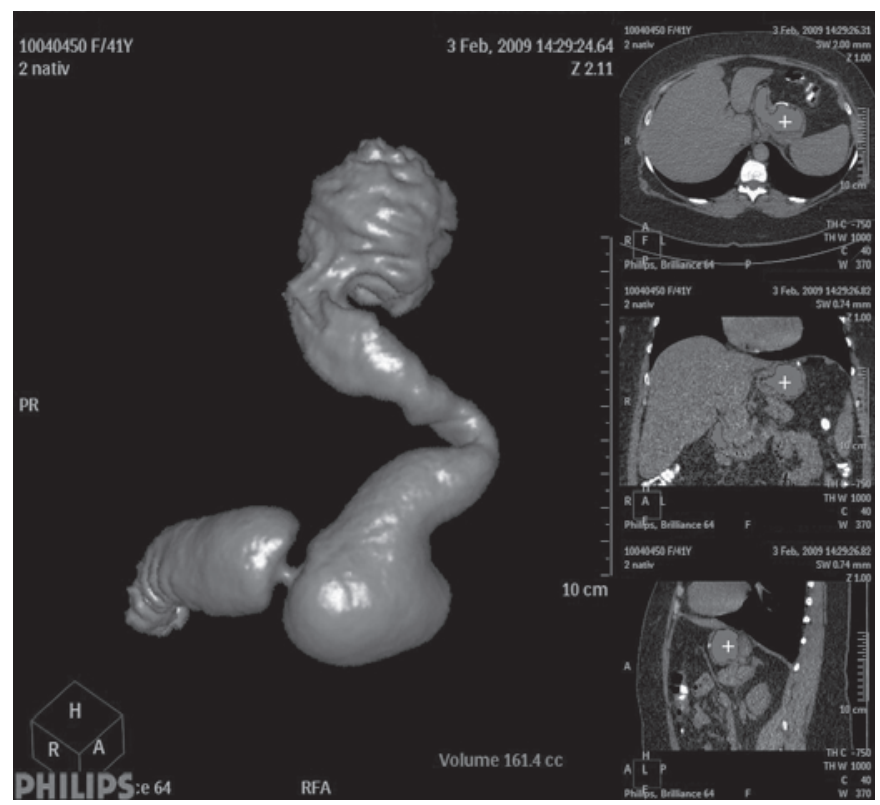

Fig. 4. Virtual CT after sleeve gastrectomy: surgical mistake with fundus in placed (uncompleted resection).

the other 2 patients, it had a central location. The high stenosis required endoscopic dilatation and stent placement; one of the central stenoses was resolved by gastric bypass cutting the stomach proximal to the stricture; the other central stenosis was resolved by adhesiolysis (fig. 6). All patients were reoperated laparoscopically. Global 30-day mortality for all LSG was $0.4 \%$. After the revisional surgery, mortality was $0 \%$.

The mean pre-revision BMI was $52 \pm 12 \mathrm{~kg} / \mathrm{m}^{2}$. The average follow-up was 49.2 months (range 3-114 months). The average BMI during follow-up was $37 \pm 8 \mathrm{~kg} / \mathrm{m}^{2}$. Laparoscopic

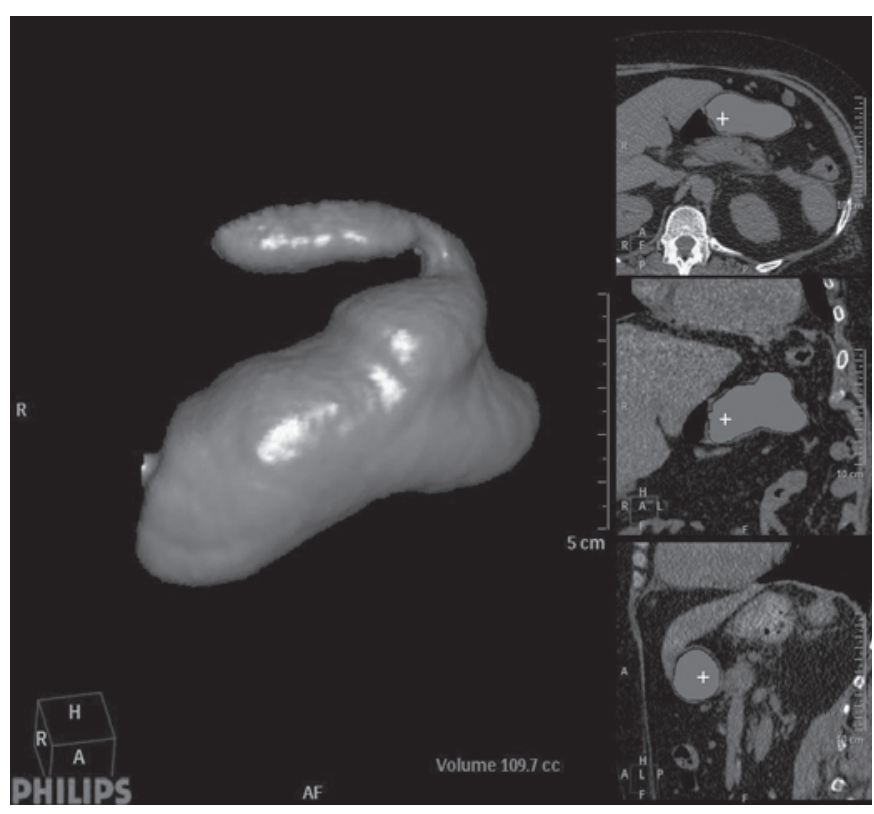

Fig. 5. Virtual CT after sleeve gastrectomy: prepyloric dilatation of the antrum 6 years after LSG.

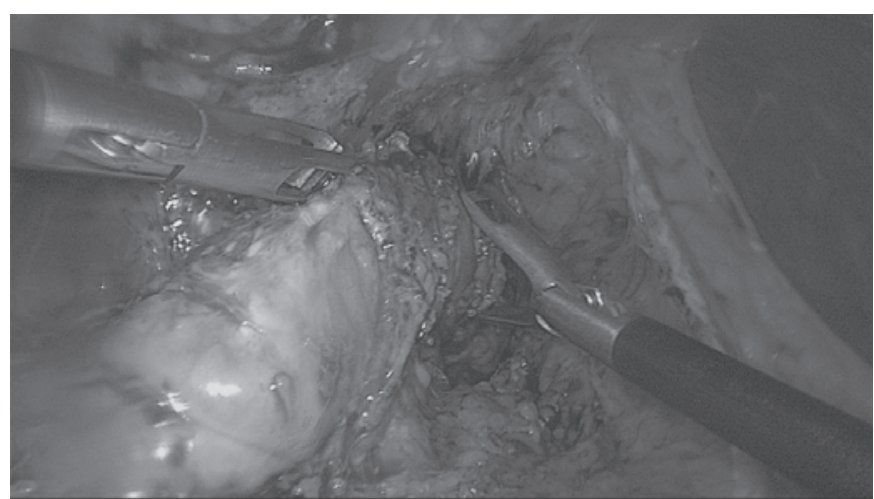

Fig. 6. Laparoscopic view on adhesiolysis and intraoperative gastroscopy for stenosis

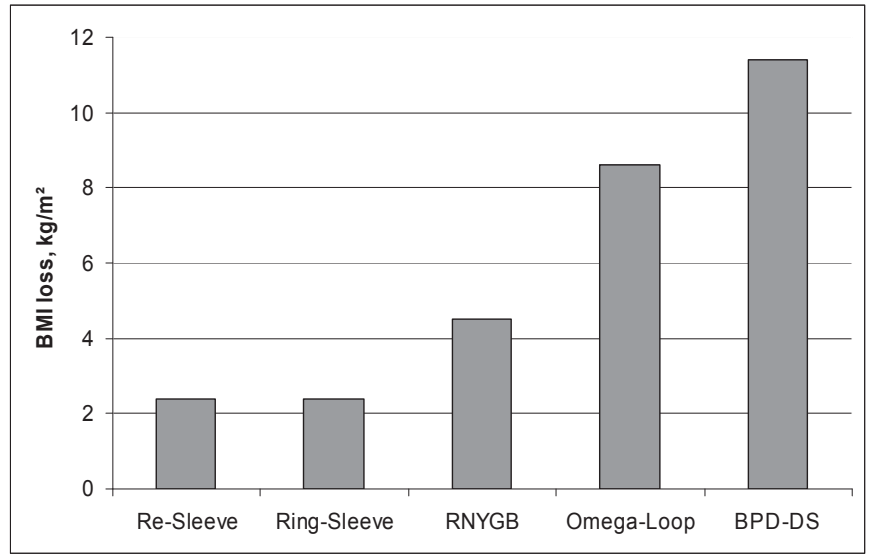

Fig. 7. BMI drop after different secondary procedures (ring placement, re-sleeve, Roux-en-Y gastric bypass (RYGB), omega-loop gastric bypass, and biliopancreatic diversion with duodenal switch (BPD-DS); BMI loss 1 and 2 years after revisional surgery. 
revision was successful in all but 1 patient who required a laparotomy for tube complications and staple line leakage. The average operating time and estimated blood loss were $82 \pm 36 \mathrm{~min}$ and $50 \mathrm{ml}$, respectively. The average hospital stay was 6.4 days. Three patients required surgical exploration for haemorrhage, staple line leakage, and an incarcerated hernia. The overall complication rate was $8.5 \%$, with a major complication rate of $5.5 \%$. None of the patients died after revisional surgery.

The postoperative weight loss varied considerably between the different types of revisional surgery. BMI loss after revisional surgery is shown in figure 7. A clear benefit of malabsorption after primary restrictive LSG is shown.

\section{Discussion}

LSG is becoming increasingly popular among obese patients and surgeons. The technique is simple, and the lack of anatomical changes regarding food ingestion makes the procedure attractive from the patients' point of view. However, in our experience, the surgical risks are higher than with banding and bypass surgery. Suture line reinforcement allows a significant reduction in bleeding but has no significant influence on the leakage rate. These finding are not new $[1,2]$, and leakage is still the Achilles heel of the procedure compared to gastric bypass surgery. Weight loss and comorbidities during the first 2 years are comparable to proximal gastric bypass, as is the problem of weight regain. There are 2 mechanisms which are responsible for this common effect: loss of restriction and/or changes in eating behaviour. In patients with proximal 'unbanded' gastric bypass, dilatation of the gastroenterostomy and the alimentary limb can be observed. The upper jejunum becomes a new reservoir (stomach). A silicon ring [3, 4] can help to prevent these changes but impairs quality of life and stimulates changes in eating behaviour to soft or liquid high caloric foods; also the procedures carries a risk of ring migration.

What is the background of the failure of the primary treatment or secondary weight regain? Our analysis has shown that approximately $50 \%$ of primary treatment failure (poor weight loss from the beginning) were caused by the surgeon (fig. 4). Incomplete resection proximally, distally, or overall was seen in our series of 285 virtual pouchographies. Generally, imaging of pitfalls gives a guideline towards the right management $[5,6]$.

In cases of weight regain after successful weight loss, we observed that the antrum area becomes a new reservoir within 2-4 years if the starting point of the resection was 4-6 cm from the pylorus. In cases with antrum resection, we observed severe reflux symptomatology with a high incidence of secondary bypass surgery. The fact that LSG is an irreversible bariatric operation is another major weakness of the procedure, at least until definitive results regarding its efficacy are obtained. Furthermore, LSG has been reported to carry a risk of GERD, perhaps because of alterations to the anatomy of the angle of His [2]. Therefore, in morbidly obese patients with pre-existing GERD, LSG should be applied with caution.

If we look at the efficacy of the second step, than we can conclude: Surgical mistakes can be successfully corrected by re-sleeve for which this is the only indication; Increased gastric volume due to antrum dilatation should not be treated by antrum resection as this may trigger GERD and potentially necessitate a third procedure; GERD after LSG can be treated very effectively by RYGB; Second procedures should only be considered if weight regain after LSG is more than $50 \%$ EWL and severe diseases such as diabetes mellitus type 2 are present; Weight regain of $15 \mathrm{~kg}$ is no indication for major abdominal surgery; Weight regain after LSG caused by changed eating behavior should be treated by adding malabsorption; Omega-loop gastric bypass causes malabsorption of fat (mild steatorrhea) and is technical the simplest procedure.

\section{Conclusions}

LSG is a safe and effective treatment for morbid obesity at mid-term follow-up. LSG is effective for comorbidity resolution, especially for the treatment of diabetes. Suture line reinforcement allows a significant reduction in bleeding. Laparoscopic revisional bariatric surgery after LSG is technically challenging but compares well in terms of safety and efficacy with open revisional procedures. Intraoperative endoscopy is a key component in performing these procedures. After failure of restriction, the addition of malnutrition (BPD-DS, omega-loop gastric bypass) seems to be the best solution for long-lasting weight loss. For GERD after LSG, RYGB is the most effective procedure at the moment. Regarding the efficacy of the second step, surgical mistakes can be successfully corrected by re-sleeve; increased gastric volume due to antrum dilatation should not be treated by antrum resection to avoid GERD; and GERD after LSG can be treated by RYGB. Second procedures should only be considered if weight regain after LSG is more than $50 \%$ EWL or severe diseases such as diabetes mellitus type 2 are present. Weight regain after LSG caused by changed eating behavior should be treated by adding malabsorption, with omega-loop gastric bypass being the simplest procedure.

\section{Disclosure Statement}

The authors declared no conflict of interest. 


\section{References}

1 Daskalakis M, Berdan Y, Theodoridou S, Weigand G, Weiner RA: Impact of surgeon experience and buttress material on postoperative complications after laparoscopic sleeve gastrectomy. Surg Endosc 2011;25:88-97.

2 Himpens J, Dapri G, Cadière GB: A prospective randomized study between laparoscopic gastric banding and laparoscopic isolated sleeve gastrectomy: results after 1 and 3 years. Obes Surg 2006;16: 1450-1456.
Karcz WK, Marjanovic G, Grueneberger J, Baumann T, Bukhari W, Krawczykowski D, Kuesters S: banded sleeve gastrectomy using the GABP ring - surgical technique. Obes Facts 2011;4:77-80.

4 Weiner RA, Weiner S, Pomhoff I, Jacobi C, Makarewicz W, Weigand G: Laparoscopic sleeve gastrectomy - influence of sleeve size and resected gastric volume. Obes Surg 2007;17:1297-1305
5 Triantafyllidis G, Lazoura O, Sioka E, Tzovaras G, Antoniou A, Vassiou K, Zacharoulis D: Anatomy and complications following laparoscopic sleeve gastrectomy: radiological evaluation and imaging pitfalls. Obes Surg 2011;21:473-478.

6 Zundel N, Hernandez JD: Revisional surgery after restrictive procedures for morbid obesity. Surg Laparosc Endosc Percutan Tech 2010;20:338-343. 